\title{
Treament of Tibial Plateau Schatzker Type VI Fractures with the Ilizarov Technique Using Ring External Fixators Across the Knee: A Retrospective Review
}

\author{
IR Ranatunga, MS Ortho (UM), M Thirumal, FRCS (Edin) \\ Department of Orthopaedics, Tengku Ampuan Rahimah Hospital Kelang, Malaysia
}

\begin{abstract}
This is a retrospective review of 18 patients with tibial plateau fractures Schatzker type VI. These cases were performed in our medical centre from January 2003 to December 2004. The Ilizarov technique (Russian technique) using the ring external fixator was the technique performed. Articular reconstruction and joint alignment were achieved with traction and olive wires with washers. One case required percutaneous elevation of the articular surface and bone grafting through a cortical window. Average clinical union was achieved within 4 months of trauma. Mean final knee flexion was $85^{\circ}$, which is compatible with walking. All patients successfully returned to their previous occupations. In conclusion, the Ilizarov technique has been shown to be a good option and a viable tool in the management of these complex and unstable injuries.
\end{abstract}

Key Words:

Knee Spanning External Fixator

\section{INTRODUCTION}

Schatzker et al. ${ }^{1}$ described a systematic classification of six types of tibial plateau fractures that was based on anteroposterior tibial plateau radiographs. These six types include pure cleavage fractures with depression, pure central depression, fractures of the medial condyle, bicondylar fractures and fractures with dissociation of the tibial metaphysis and diaphysis. Schatzker type VI tibial plateau fracture truly encompassed transverse or oblique fractures of the proximal third of the tibia with a fracture of one or both tibial condyles and articular surfaces and a dissociation of the metaphysis and diaphysis regions. Agnew et al. ${ }^{2}$ reported in his study that out of 2440 reported tibial plateau fractures there were only reported 41 cases of Schatzker type VI fractures. The rarity of this type of fracture has caused very limited documentation of the treatment. Most of the papers published have advocated operative intervention ${ }^{3}$.
Schatzker type VI tibial plateau fractures are caused by considerable high energy trauma. This traumatic force not only causes severe bony comminution but also considerable soft tissue injury. Watson et al. ${ }^{4}$. described these high energy injuries as those which cause significant articular depression, condylar displacement, metadiaphyseal fracture extension with open wounds or extensive closed degloving injuries of the proximal tibia. Complications include severe soft tissue compromise requiring coverage procedures, lower limb compartment syndrome, peroneal nerve injury, vascular injury and eventual knee arthrosis ${ }^{5}$. If the soft tissue injuries are not adequately treated, amputation may be necessary in the end. Associated injuries include ipsilateral femoral and tibial diaphyseal fractures, cruciate and collateral ligament injuries and meniscal tears ${ }^{6}$. All these complications directly impact surgical treatment and finally long term outcomes.

The goals of treatment are anatomical reconstruction of the articular surface, restoration of the limb axis, fixation spanning the metaphyseal comminution and further minimization of morbidity to an already traumatized soft tissue envelope ${ }^{7}$. These objectives can be achieved with internal fixation, bridge plating, percutaneous screws with casting, external fixators with or without limited open and bone grafting or a combination of these methods.

In the 1990s, the concept of 'spanning' the knee joint was introduced. This concept evolved as proponents of indirect fracture reduction and biological fixation reported increased rates of fracture healing with decreasing infection rates in this type of fracture ${ }^{8}$. With this method, the fractures are indirectly reduced with traction, and then maintained with either internal or external fixation before a knee spanning external fixator is finally applied. Watson et al introduced the knee spanning external fixator to rest the knee joint. With the knee spanning external fixator, reduction of the intraarticular fragments is maintained.

The aim of this study was to assess the clinical outcomes of treating tibial plateau fracture type VI with Ilizarov spanning fixator across the knee joint. 


\section{MATERIALS AND METHODS}

\section{Study Sample}

This is a retrospective study of 18 patients seen at our hospital from January 2003 to December 2004. The data source was Orthopaedic operative registry book. All patients from this time period with the diagnosis of tibial plateau fractures were enrolled into study. Medical records and radiographs were studied by the authors. The patients were also seen on follow up with the Ilizarov Clinic. Four polytrauma patients were excluded. In the Ilizarov clinic the patients' outcomes were recorded including range of flexion of the knee joint and level of activity at their present occupation. The follow up period was one year post trauma. These 18 patients underwent Ilizarov technique with ring fixators spanning the knee joint. All operative procedures were performed by the senior consultant. One patient needed percutaneous elevation of the articular fragment and bone grafting. None had open reduction or screw fixation.

\section{Operative Technique}

The patient was positioned supine on a normal radiolucent table. No tourniquet was applied. The affected limb was held on two 'U' wooden supports (Figure 1), which were placed posteriorly at the thigh region and at the heel. These are useful in creating the necessary space for the application of the ring fixators. A calcaneal Steinmann pin is then inserted with weights 20 to $25 \mathrm{~kg}$ weights across a Mayo table (Figure 1) to achieve ligamentotaxis.

The first $1.8 \mathrm{~mm}$ olive wire with a $3.5 \mathrm{~mm}$ washer was inserted using image intensifier in a lateral to medial direction just anterior to the head of the fibula at the tibial plateau subchondral region to provide interfragmentary compression (Figure 2). The size of the ring needs to have at least 2 fingerbreadths clearance from the patient's skin circumferentially and is fixed to the first wire with two centre fixation bolts and tensioned to 110 Newton to create compression in between the articular fragments thus reconstructing the articular surface. Another olive wire is applied from medial to lateral on the distal side of the ring (Figure 3) and then a drop wire is inserted.

This ring with three tensioned wires is then connected to two more completed rings distally with three interconnecting rods (Figure 3) at the midshaft of the tibia with the most distal ring at the distal metaphysic of the tibial bone. Both rings are attached to the tibia with one Schanz pin and one tensioned K-wire. The construct is then completed with a half ring attached to the femur on the lateral side with two Schanz pins attaching it to the femoral condyle. This femoral half ring is attached to the proximal first complete ring with two interconnecting rods spanning the knee joint in extension thus maintaining ligamentotaxis. The weights on the traction are then released.
There was no screw fixation, nor was there was arthroscopically assisted reduction. One patient needed percutaneous elevation of the articular fragments and bone grafting through a cortical bone window.

\section{Postoperative}

All patients were allowed to weight-bear as tolerated. Some found it difficult to weight-bear because of the knee spanning ring fixator. The femoral half ring was removed six weeks postoperatively. After the removal of the femoral half ring, patients were encouraged to fully weight-bear. At twelve weeks, the interconnecting threaded rods at the fracture site were removed to test clinical union. If there was any abnormal motion at the fracture site the fixator was not removed and the threaded rods were reconnected again. If the fracture was clinically united then the fixator was completely removed. Follow-ups were done at 2, 4, 6, 8, 12, 16, 20 weeks, 6 months and the final follow up visit was at one year post trauma.

Radiographic examination (Figure 4) and knee range of movements were recorded. The time of radiographic union was recorded. Radiographic union was achieved when three out of four cortices on anteroposterior and lateral radiographs showed bony continuity. There were no secondary surgeries performed. Local pin site care was instituted with water and soap. The patients were encouraged to wash the pin sites and the frame at least three times a day.

\section{RESULTS}

The study sample was comprised of three females and fifteen males patients. There were twelve Malays and six Indians. Sixteen patients between 20 to 50 years old and two patients were above 50 years. There were three open fractures; one case was a Grade 1 open fracture and two cases were Grade 2 open fractures. The rest were closed fractures. The mean interval between the injury and application of the Ilizarov ring external fixator was 7.34 days in our hospital.

Fractures were considered united once radiographic union was achieved and there was no abnormal motion, which occurs with stressing of the fracture site after loosening of the frame.

Mean union time was recorded at 3.72 months. All fractures were united within four months. Ten patients required additional casting and eight patients did not require any other form of supports.

Mean flexion of the affected knee joint was $85^{\circ}$ at final follow up at one year post trauma. Three patients had flexion knee contracture of $5^{\circ}$. Nine out of 18 cases $(50 \%)$ had range of motion $80^{\circ}$ or less, and 5 out of 18 cases $(27.8 \%$ ) had flexion $60^{\circ}$ or less. 
Table I: Physical outcome of surgery and complications

\begin{tabular}{|c|c|c|c|c|c|c|}
\hline NO. & DIAGNOSIS & $\begin{array}{l}\text { INTERVAL } \\
\text { (days) }\end{array}$ & $\begin{array}{l}\text { UNION } \\
\text { (months) }\end{array}$ & $\begin{array}{l}\text { RANGE OF } \\
\text { MOTIONS( }\left(^{\circ}\right)\end{array}$ & COMPLICATIONS & REMARKS \\
\hline 1 & Closed Fractures & 5 & 3 & $0-30$ & Pin site infection & $\begin{array}{l}\text { Infection treated with } 1 \text { week of } \\
\text { antibiotics \& dressings }\end{array}$ \\
\hline 2 & Closed Fractures & 5 & 4 & $0-110$ & & \\
\hline 3 & Closed Fractures & 10 & 4 & $5-60$ & & Medical co-morbidities \\
\hline 4 & Closed Fractures & 11 & 3 & $0-60$ & & Medical co-morbidities \\
\hline 5 & Closed Fractures & 7 & 4 & $0-100$ & & \\
\hline 6 & Closed Fractures & 13 & 4 & $0-100$ & & \\
\hline 7 & Closed Fractures & 4 & 5 & $5-80$ & & \\
\hline 8 & Closed Fractures & 7 & 3 & $0-100$ & & \\
\hline 9 & Closed Fractures & 14 & 4 & $0-100$ & & Medical co-morbidities \\
\hline 10 & Open Fractures & Same day & 4 & $0-80$ & Pin site infection & $\begin{array}{l}\text { Infection treated with } 1 \text { week of } \\
\text { antibiotics \& dressings }\end{array}$ \\
\hline 11 & Open Fractures & Same day & 4 & $0-110$ & & \\
\hline 12 & Open Fractures & Same day & 3 & $0-110$ & & \\
\hline 13 & Closed Fractures & 15 & 2 & $0-110$ & & Medical co-morbidities \\
\hline 14 & Closed Fractures & 15 & 4 & $0-60$ & Pin site infection & $\begin{array}{l}\text { Infection treated with } 1 \text { week of } \\
\text { antibiotics \& dressings } \\
\text { Medical co-morbidities }\end{array}$ \\
\hline 15 & Closed Fractures & 4 & 5 & $0-60$ & & \\
\hline 16 & Closed Fractures & 6 & 3 & $5-110$ & & \\
\hline 17 & Closed Fractures & 5 & 5 & $0-80$ & Pin site infection & $\begin{array}{l}\text { Infection treated with } 1 \text { week of } \\
\text { antibiotics \& dressings }\end{array}$ \\
\hline 18 & Closed Fractures & 12 & 3 & $0-80$ & & Medical co-morbidities \\
\hline
\end{tabular}

Four patients had pin site infection. These infections were treated with a one-week course of antibiotics and daily dressings. There were no other recorded complications of septic arthritis, osteomyelitis, amputation or knee laxity. All the patients were allowed to go back to work within 5 months of their injury and were able to carry out the requirements of their job level occupation as before the injury.

\section{DISCUSSION}

Tibial plateau Schatzker type VI energy fractures, caused by high energy trauma, inflict extensive damage to the bone and more injury to the soft tissue. Early problems in management of this high energy complex fracture include fracture instability and inability to internally fixate to address the stability when open reduction is attempted. Postoperative complications include infection, wound dehiscence and wound healing problems ${ }^{9}$. Long term complication include secondary knee osteoarthritis, loss of reduction, collapse of articular fragments, malunion and nonunion ${ }^{10}$. Dissociation of the metaphysis from the diaphysis does not allow for conservative treatment with traction. It has been shown that long term immobilization can cause decreased function of the knee joint ${ }^{11}$.

We have shown in the present study, that despite extensive articular comminution and spanning of the fixator across the knee joint, satisfactory functional results can be achieved with the Ilizarov frame. Reduction of intraarticular fragments is confirmed indirectly by the image intensifier. Opponents of this technique may argue that congruous reduction can only be confirmed by open reduction, but even with open reduction one requires imaging to confirm congruous reduction.

Internal fixation of these highly complex injuries had lead to some disastrous results. Moore and Harvey ${ }^{12}$ reported 23\% of cases treated with open reduction with internal fixation (ORIF) became infected. The rate of infection with ORIF reported by Malik et al. was four out of five ${ }^{13}$, Young and Barrack report seven out of eight ${ }^{14}$ and Yang et al. ${ }^{15}$ reported five out of sixteen for type VI fracture.

Morandi et al. reported an advantage of external fixation of complex tibial plateau fractures was decreasing rates of complications ${ }^{16}$. Marsh et al. in his paper advocated the 'spanning' concept across the knee joint to allow the knee joint to rest and further immobilize the articular fragments ${ }^{17}$. In our series, we saw that the optimal fixation time was six weeks to give enough time for the intra-articular fractures to form soft callus. Spanning the knee with the external fixators also prevents wire migration into the knee joint and the associated risk of septic arthritis.

The six-week period of keeping the knee in extension caused some stiffness, but the mean flexion of the affected knee joint was satisfactory was $85^{\circ}$ at final follow up at one year post trauma. There were three patients who had flexion knee contracture of $5^{\circ}$. Nine out of eighteen cases $(50 \%)$ had an average flexion of $80^{\circ}$ or less, and five out of eighteen cases $(27.8 \%)$ had an average flexion of $60^{\circ}$ or less. According to Ali et al., flexion of less than $60^{\circ}$ is incompatible with normal gait ${ }^{18}$. 


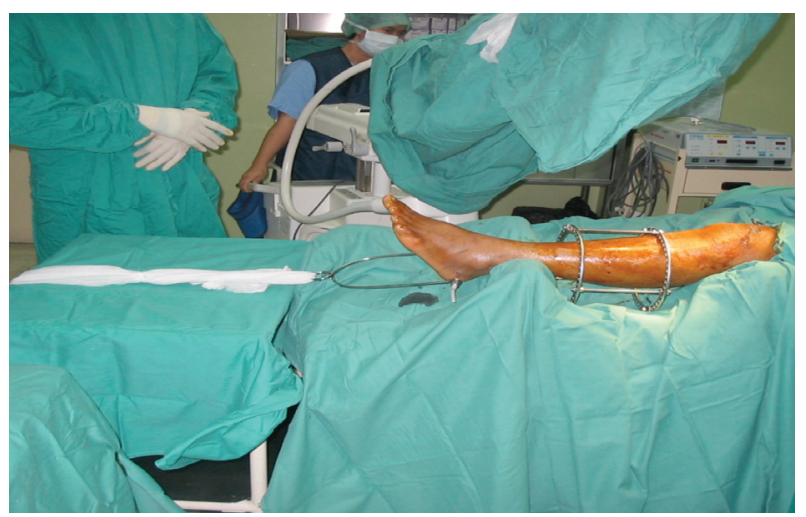

Fig. 1: Patient is in supine position with the affected limb on the draped ' $\mathrm{U}$ ' supports with traction across the Mayo table.
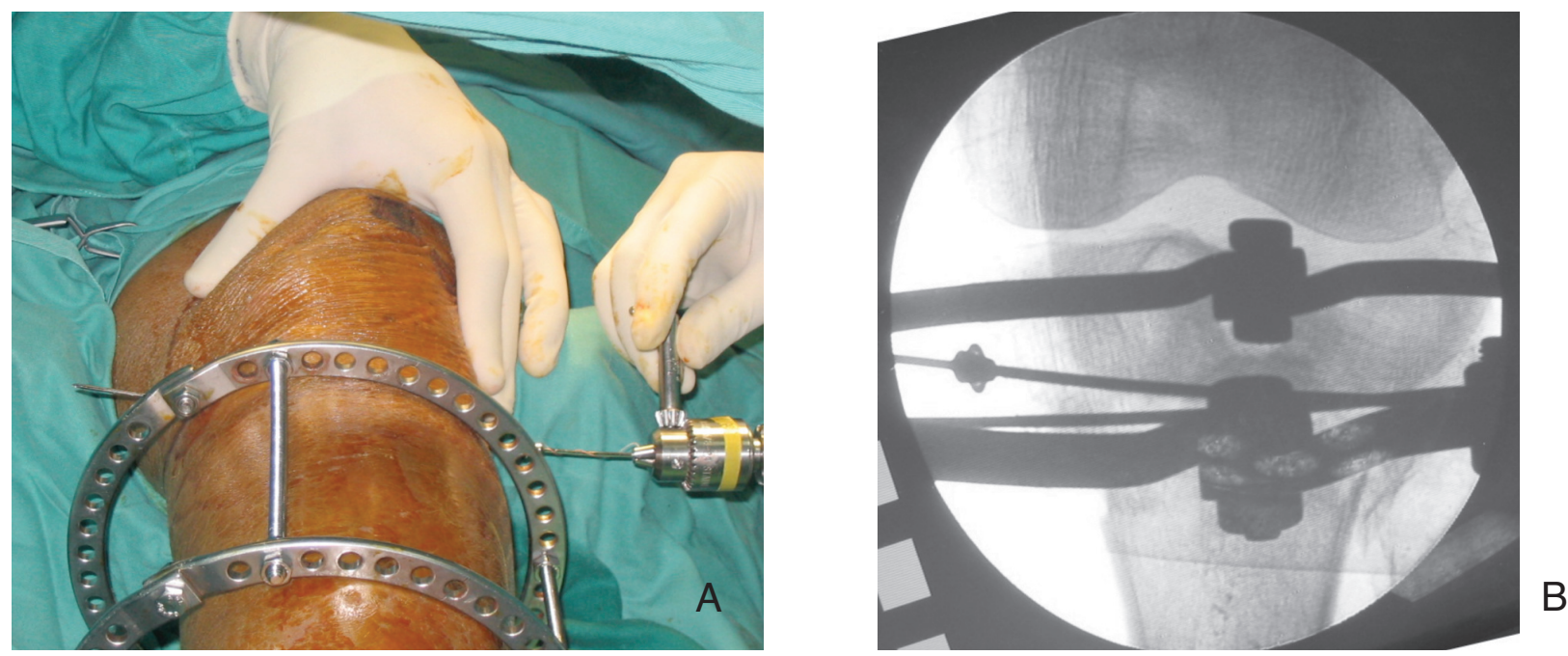

Fig. 2: A parallel K-wire to the joint line is inserted (a) under guidance of the image intensifier (b).

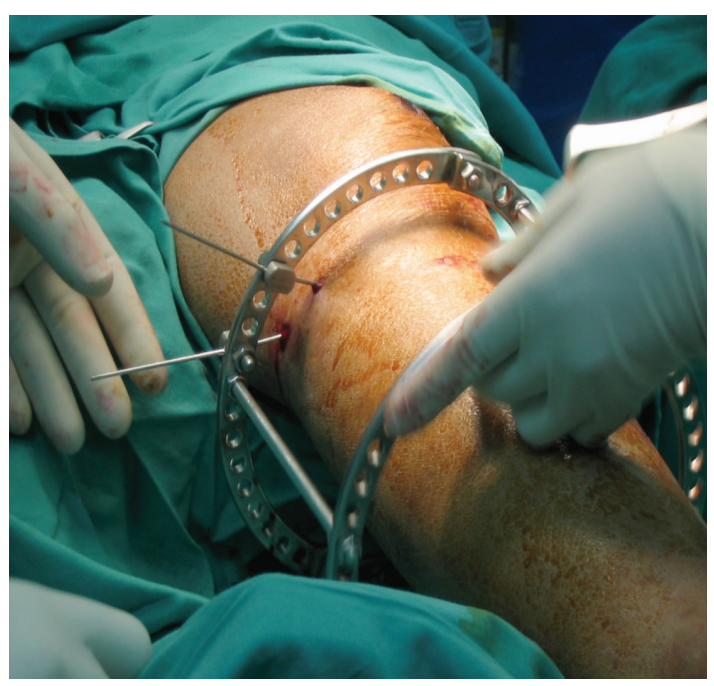

Fig. 3: Insertion of two more wires to stabilize the proximal ring. 

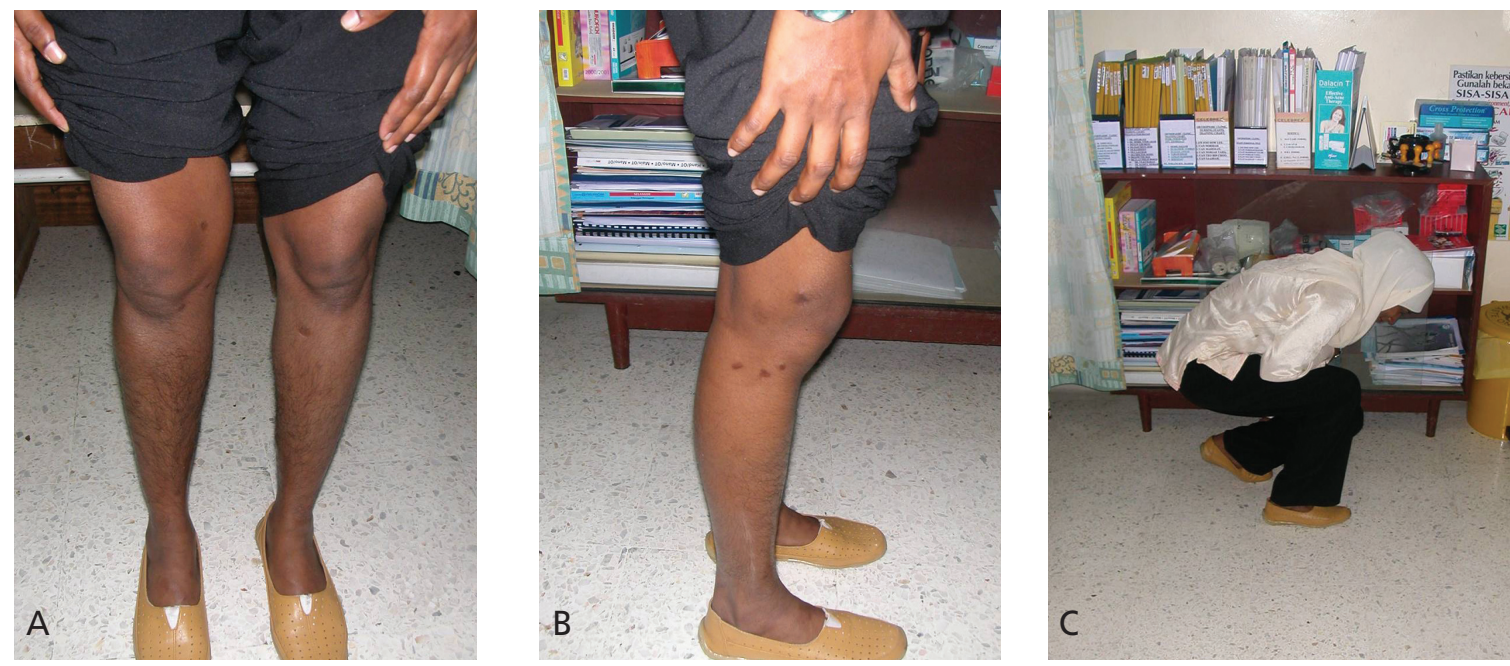

Fig. 4: Example of a patient with tibial plateau fracture type $\mathrm{VI}$ after treatment (A). Her knee extension (B) and flexion (C) are shown.

In the current study, most patients were able to walk and achieved functional knee flexion which allowed them to go back to their original occupation.

The open fractures were fixated immediately as an emergency procedure, but closed fractures were treated electively after the comorbidities were optimized. The differences in when the operative procedure was performed did not reflect the final knee range of movement. There were patients on whom the operative procedures were performed 10 days after the trauma range of motion of $110^{\circ}$ at the one year follow up. In these cases, the ring fixator was extended up to the femoral shaft for six weeks. The femoral half ring was then removed and the patient was encouraged to weightbear to encourage more healing. We did not have any complications of deep infection, osteomyelitis or knee laxity, since our technique was performed percutaneously.

Among the drawbacks of the Ilizarov technique are the risk of losing full range of movement of the knee joint and the visually less appealing and cumbersome ring external fixator. In addition, it is also a very demanding technique with a steep learning curve, requiring specialized training and experience. Hence it has not gained popularity to date even though it has been practiced for more than 15 years in this country with promising results.
The limited size of the study sample was a limiting factor of this study. It is our aim to increase the awareness that knee spanning ring fixation is a safe and viable technique for treatment of these highly unstable injuries. In addition, this study also did not explore the joint surface thoroughly with a computerized tomography study or arthroscopic examination of the joint to look for joint incongruity. Therefore, a long term outcome study should be undertaken to examine the development of secondary onset of posttraumatic knee osteoarthritis in this group of patients.

\section{CONCLUSION}

We believe that ring fixation with small K-wires and olive wires with a temporary spanning knee half ring is efficacious for treating both the bony articular comminution and soft tissue damage. Intraoperative traction and the insertion of small K-wires to stabilize the articular fragments are essential to reestablishing the alignment and the length of the tibial bone. The external circular rings attach these K-wires and Schanz pins to a rigid $360^{\circ}$ frame which neutralizes all shear and rotational forces. Early weight bearing as tolerated after removal of the femoral ring especially after six weeks does not disturb knee function and also encourages fracture healing by axial micromotion without shear force ${ }^{19}$. Subsequently, all patients had bony union within four months and all returned to their work within six months. Therefore, we have adopted the Ilizarov method for all open or closed Schatzker type VI tibial plateau fractures. 


\section{REFERENCES}

1. Schtzker J, McBroom R, Bruce D. The tibial plateau fracture: the Toronto experience 1968-75. Clin Orthop 1979; 138: $94-104$.

2. Agnew SG, Bernirschke SK, Mayo KA, Henley MB, Santaro VM. Open reduction and internal fixation of complex tibial plateau fractures. J.Orthop Trauma 1991; 5: 236.

3. Mikulak, Steve A, Gold, Stuart M, Zinar, Daniel M. Small wire external fixation of high energy tibial plateau fractures. J Orthop Trauma 1998; 356: 230-8

4. Watson JT. High energy fractures of the tibial plateau. Orthop Clin North Am 1994; 25: 723-52.

5. Honkonen SE. Degenerative arthritis after tibial plateau fractures. J Orthop Trauma 1995; 9: 273-7.

6. Bennett WF, Browner B. Tibial plateau fractures: A study of associated soft tissue injuries. J Orthop Trauma 1994; 8:183-8.

7. William JM, Sean EN. Open reduction and internal fixation of high energy tibial plateau fractures. Orthop Clin North Am 2002; 33: 177 .

8. Leunig M, Hertel R, Siebenrock KA. The evolution of indirect reduction techniques for the treatment of fracture. Clin Orthop 2000; 375: 7-14.

9. Moore TM, Patzakis MJ, Harvey JP. Tibial plateau fractures: definition, demographics, treatment rationale and long term results of closed traction management or operative reduction. J Orthop trauma 1987; 1: 97-119.

10. Waddell JP, Johnston DWC, Neidre A. Fracture of the tibial plateau: a review of ninety-five patients and comparison of treatment methods. J Trauma 1981; 21: 376-81.

11. Gausewitz S, Hohl M. The significance of early motion in the treatment of tibial plateau fractures. Clin Orthop 1988; 202 : $135-8$.

12. Moore TM, Patzakis MJ, Harvey JP. Tibial plateau fractures: definition, demographics, treatment rationale and long term results of closed traction management or operative reduction. J Orthop trauma 1987; 1: 97-119.

13. Malik AR, Covall DJ, Whitelaw GP. Internal versus external fixation of bicondylar tibial plateau fractures. Orthop Rev 1992; 21: 1443-36.

14. Young MJ, Barrack RL. Complications of internal fixation of tibial plateau fractures. Orthop Rev 1994; 23: 149-54.

15. Yang EC, Weiner L, Strauss E, et al. Metaphyseal dissociation fractures of proximal tibia: An analysis of treatment and complications. Am J Orthop 1995; 24: 695-705.

16. Morandi M, Watson JT, Blake R, et al. Treatment of complex tibial plateau fractures with circular external fixators. Orthop Trans 1993-1994; 17: 1056.

17. Marsh JL, Smith S, Do TT. External fixation and limited internal fixation for complex fractures. J Bone Joint Surg (Am) 1995; 77: 661-3.

18. Ali AM et al. Clin Orthop Relat Res 2004; 415: 214-5.

19. Kershaw CJ, Cunningham JL, Kenwright J. Tibial external fixation, weight bearing and fracture movement. Clin Orthop 1993; 293: 28-36. 